\title{
Saúde da Família, agora mais do que nunca! *
}

\author{
Family H ealth, more than never!
}

Claunara Schilling M endonça ${ }^{1}$

\footnotetext{
* Segundo o relatório da Organização M undial de Saúde de2008, "Primary $\mathrm{H}$ ealth Care, now more than ever".

${ }^{1}$ Departamento deAtenção Básica. Secretaria de Atenção à Saúde. M inistério da Saúde. Esplanada dos M inistérios, Bloco G, 655. 70058-900

Brasília DF.

claunara@saúde.gov.br
}

\begin{abstract}
The implementation of Primary $\mathrm{H}$ ealth Care as a strategy for organizing health care is undoubtedly important. There are already sufficient evidences that countries whose health systems are organized from the principles of Primary Care achieve better results on health with lower costs, higher level of user satisfaction and greater equity even in situations of great social inequity such as in Brazil. Strengthening Primary Care in Brazil has been a gradual process, showed with the expansion of the coverage of Family H ealth teams in all states of the federation, nowadays wehave 29,148 of family health teams, 228,412 community health workers and 17,588 oral health teams working throughout the country. There are multiple studies on Family $\mathrm{H}$ ealth Strategy showing positive results that let no doubt about the success of this strategy. Despite all the advances, challenges were placed ahead, the most important are: the challenge of training and permanent education, the challenge of consolidating the PHC as system's coordinator and the challenge of communicating with society. Weare on track, meet the challenges mentioned is an obligation of Brazilian society in the maintenance of greater social inclusion's policy, which is the U nified Health System driven by the primary care/Family Health Strategy.

Key words Primary Health Care, Family Heath Strategy, U nified Health System
\end{abstract}

Resumo A implantação da atenção primária como uma estratégia de organização dos sistemas de saúde tem relevância inquestionável. Já existem evidências suficientes que os países cujos sistemas de saúde se organizam a partir dos princípios da atenção primária alcançam melhores resultados em saúde com menores custos, maior satisfação dos usuários e maior equidade, mesmo em situaç̃os de grande desigualdade social, como éo caso do Brasil. 0 fortalecimento da atenção primária no Brasil tem sido um processo gradativo, traduzido no aumento de cobertura das equipes de Saúde da Família em todos os estados da federação, sendo que hoje temos 29.678 equipes de saúde da família, 229.572 agentes comunitários e 18.220 equipes de saúde bucal atuando em todo o país. Os resultados positivos nas avaliações da Saúdeda Família são múltiplosenão deixam dúvida do sucesso da estratégia. A pesar de todos os avanços, alguns desafios estão postos para o futuro; entre eles, destacam-se o desafio da formação e educação permanente, o desafio de consolidar a atenção primária à saúde(APS) como ordenadora do sistema eo desafio da comunicação com a sociedade. Estamos no caminho certo, vencer os desafios apontados é obrigação da sociedade brasileira na manutenção de sua maior política de inclusão social, que é o Sistema Ú nico de Saúde orientado pela atenção primária/ Saúde da Família.

Palavras-chave Atenção Primária à Saúde, Saúde da Família, Sistema Ú nico deSaúde 
2008, ano marcado por celebrações. No mundo, os trinta anos deAlma-Ata, no Brasil, outros dois grandes e importantes aniversários: os vinte anos do SistemaÚ nico de Saúde eos quinze da política brasileira de atenção primária - a Saúde da Família.

Quando inicia o Programa dos Agentes Comunitários de Saúde, em 1991, o M inistério da Saúde reconhecia experiências exitosas quejávinham acontecendo nos municípios brasileiros, em alguns casos antes mesmo da própria criação do SUS.

Em dezembro de 1993, decide-sepela criação do Programa de Saúde da Família, também baseado em experiências municipaise, por ser uma nova forma de abordar a atenção primária em saúde no Brasil, começou lentamente a ser implantado nos municípios brasileiros, notadamente em algumas áreas do nordeste emunicípios menores no interior do Brasil. O M inistério da Saúde estimula efomenta, com a criação do Piso de Aten ção Básica (PAB), em 1998, recursos perenes de forma per capita, a todos os municípios brasileiros, bem como garante recursos específicos para o Programa de Saúde da Família e dosagentes comunitários de saúde. O PAB altera a modalidadeem vigor no SUS de pagamento mediante produção de serviços, representando uma importante inovação no modelo de financiamento da saúde, pois inicia o repasse, pela primeira vez no país, de recursos federais de forma mais equitativa, a todos os municípios brasileiros. A criação do PAB variável da Saúde da Família acelerou o ritmo de expansão, com a adesão de muitos municípios a esta estratégia, e permitiu o acesso de centenas de brasileiros aos serviços de saúde.

A implantação da atenção primária como uma estratégia de organização dos sistemas de saúde tem relevância inquestionável. Já existem evidências suficientes que os países cujos sistemas de saúde se organizam a partir dos princípios da atenção primária alcançam melhores resultados em saúde, menores custos, maior satisfação dos usuários e maior equidade, mesmo em situações de grande desigualdade social, como é o caso do Brasil.

A APS brasileira está definida como um conjunto de ações de saúde no âmbito individual e coletivo, que abrangem a promoção e proteção de saúde, prevenção de agravos, diagnóstico, tratamento, reabilitação e manutenção da saúde. Desenvolve-se por meio de práticas gerenciais e sanitárias democráticas e participativas, sob forma de trabalho em equipe, destinadas a populações de territórios geograficamente delimitados, pelasquais assume a responsabilidade sanitária, considerando a dinâmica existente no território onde vivem essas populações. Sendo o contato preferencial dos usuários com o sistema de saúde, se orienta pelos princípios da universalidade, acessibilidade e coordenação, vínculo e continuidade, integração, responsabilidade, humanização, equidade e participação social ${ }^{1}$.

Esse conceito evidencia que estamos construindo, no sistema de saúde brasileiro, uma atenção primária à saúde integral, ampla e abrangente, e não a APS sel etiva, de pacotes básicos para pobres.

É importante lembrar que os anos noventa, quando se inclui milhões de brasilei ros no sistema de saúde, optamos por um processo incremental dedescentralização emunicipalização, enquanto 0 mundo vivia o momento de liberalização econômica, com o Consenso de Washington, cujos pilares 0 ano de 2008 também, de forma emblemática, tem demonstrado suas fraturas.

Desde Alma-Ata, a atenção primária em seu conceito de orientadora dos sistemas de saúde, principalmente os de caráter universal, foi implementada nos países ricos como Inglaterra, Espanha, Suécia, Holanda, Canadá, N ova Zelândia, entre outros, enquanto a experiência dos países em desenvolvimento, principalmente na América Latina, foi de um modelo seletivo, de uma atenção para pobres.

Temos claro no Brasil que os pacotes focalizados não cabem em nossa proposta de SUS, mas não esquecemos as populações que historicamente foram negligenciadas pela sociedadebrasileira. Por isso temos incentivos especiais dentro da atenção primária para atendimento a populações quilombolas, aos assentamentos agrários, às populações indígenas ea todos os municípios do país com baixo Índice de Desenvolvimento Humano (IDH). Estamos assim promovendo equidade na distribuição dos recursos da atenção primária, inclusive para compensar a desigualdade de distribuição histórica da capacidade instalada de serviços hospitalares, baseada quase que exclusivamente na oferta dos mesmos.

Países como o Brasil e outros de ren da intermediária têm que estar atentos à necessidade que os sistemas de saúde têm em regular a incorporação tecnológica, abusiva e acrítica, essencial para a sustentabilidade de nossas propostas de sistemas universais de saúde.

O fortalecimento da atenção primária no Brasil tem sido um processo gradativo, com 0 aumento de cobertura das equipes de Saúde da Família em todos os estados da federação. U m esforço tripartite que superou as divergências políticopartidárias para avançar na implantação dos preceitos constitucionais.

O caráter estruturante dos sistemas municipais de saúde orientados a partir da Estratégia Saúde da Família tem provocado um importante mo- 
vimento de reordenamento do modelo de atenção no SUS. A estratégia busca cumprir os princípios da APS: ser o primeiro contato da população às ações e serviços de saúde, com integralidade, ao longo do tempo e coordenando os usuários na rede de serviços². Dessa forma, pressupõe maior racionalidade na utilização dos demais níveis assistenciais. Os resultados positivos nas avaliações da Saúde da Família se referem à avaliação dos usuários, dos gestores e dos profissionais de saúde3, à oferta de ações de saúde e ao acesso e uso de serviços ${ }^{4,5}$ e à redução da mortalidade infantil ${ }^{6}$.

0 exemplo da redução da mortalidade infantil nas duas últimas décadas, fazendo com que o Brasil apresentasse o melhor desempenho no grupo entre os países analisados para 0 al cance dos $\mathrm{Ob}$ jetivos de Desenvolvimento do Milênio (ODM), é creditado a dois importantes programas sociais: Saúdeda Família, em especial ao trabalho dos agentes comunitários de saúde, e ao Bolsa Família, programa de transferência direta de renda com acompanhamento de condicionalidades, que beneficia famílias em situação de pobreza, integrante do Fome Zero ${ }^{7}$.

Ainda utilizando o exemplo da redução da mortalidade infantil, um indicador clássico, mas permeado por vários dos determinantes sociais da saúde, estudo demonstrou que o aumento na cobertura deSaúdeda Família foi capaz dereduzir em 4,6\% a mortalidade infantil, controlado para acesso à água, saneamento, renda, escolaridade materna e número de filhos, número de médicos e enfermeiros, leitos hospitalares e cobertura vacinal ${ }^{6}$.

Outro estudo aponta que, nos municípios brasileiros com baixo IDH, enquanto a variação média anual da taxa de mortal idadeinfantil reduz em 4,3\% nos que têm mais de $70 \%$ de cobertura da SF, naqueles municípios com menos de $20 \%$ de cobertura, a variação média anual foi crescente em $4,5 \%{ }^{8}$.

Podemos usar outro exemplo, numa área em que o Brasil se destaca, que é da al ta capacidade de realizar programas de imunização, erradicando doenças preveníveis por imunização. A campanha da rubéola, realizada esse ano, que vacinou $58 \mathrm{mi}-$ Ihões de pessoas, representando $85 \%$ da meta, contou com a capilaridade da atenção primária no Brasil, sendo outra demonstração recente de êxito no acesso da população brasileira a uma importante ação de prevenção que visa erradicar essa doença.

Em 2006, a Política Nacional deAtenção Básica torna-se uma das prioridades inseridas no Pacto pela Vida: buscando "consolidar e qualificar a estratégia Saúde da Família como modelo de Atenção Básica e centro ordenador das redes de atenção à saúde no Sistema Ú nico de Saúde".
Em 2008, a Saúde da Família entra como um dos eixos centrais do M ais Saúde para M ais Brasileiros, projeto central do segundo governo do Presidente Lula, com uma meta de alcançar $70 \%$ da população brasileira até 2011.

A Saúde da Família hoje ultrapassou em muito os limites de um programa e é uma política do Estado brasileiro, estando na agenda dos gestores do SUS e uma prioridade da atual gestão federal, pois é parte das metas presidenciais do governo Lula.

H oje são 29.678 equipes de saúde da família, 229.572 agentes comunitários e 18.220 equipes de saúde bucal, uma especificidade do modelo brasileiro, que, parte do Brasil Sorridente, oferece a integralidade da atenção à saúde bucal.

OsNúcleos deA poio à Saúdeda Família(NASF), cuja implantação inicia em 2008 eem junho de 2009 somam 648 em funcionamento no país, representam um marco importante na ampliação das possibilidades dealcançar melhores resultados em saúde, com o enfoque na promoção da saúde e no cuidado à população. Com a inclusão dos novos profissionais de saúde, vinculados às equipes de Saúde da Família, aumenta-se a possibilidade de responder aos novos e antigos desafios da morbidade dos brasileiros. Como o sofrimento psíquico, as modificações no padrão nutricional, em que $40 \%$ dos adultos são obesos e $27 \%$ dos escolares têm sobrepeso e 0 aumento da longevidade da população, que, se por um lado, é o resultado do desenvolvimento social e econômico dos países, no nosso, o envelhecimento veio antes do crescimento econômico e resulta, para o sistema de saúde, maior número de pessoas com co-morbidades e aumento de carga de doenças. Os NASF trazem a possibilidade de ampliar a oferta das práticas integrativas e complementares e a oferta da melhor tecnologia disponível para grande parte das doenças crônicas, bem como a revisão dos tratamentos baseados somente na medicalização das doenças.

\section{D esafio da formação e educação permanente}

A meta de expansão para toda a população brasileira traz o principal desafio para os próximos anos, queéo que temos chamado de "valorização política e social da Saúde da Família". A advocacy da APS passa pela qualificação da Saúde da Família. Essa depende de mudanças estruturantes na formação - graduação e pós-graduação - das áreas da saúde. No caso da formação médica, segundo levantamento de necessidades de formação profissional para o SUS da Comissão Interministerial de Gestão da Educação na Saúde, instituída pelo Decreto 
Presidencial de 20/06/2007, se faz necessária a ampliação das vagas de residência em medicina de família e comunidade. $\mathrm{Na}$ graduação, a decisão dos alunos em se especial izarem para trabalhar na Saúde da Família depende também do "espelho" dos seus formadores, o que requer, nas universidades, mestres e doutores em APS, capazes de captar recursos, fazer pesquisas e ser modelos a serem seguidos pelos alunos.

Precisamos formar em larga escala e certificar os profissionais que fazem parte hoje da Saúde da Família, bem como os gestores do SUS, tanto nos $80 \%$ dos municípios brasileiros de pequeno porte, responsáveis no seu território pelos serviços deAPS, bem como nas regiões metropolitanas e nos grandes municípios, cuja demanda é a substituição de modelos tradicionais de APS para a Saúde da Família, como coordenadora do cuidado nas redes de atenção à saúde, proposta encaminhada pela UniversidadeA berta do SUS, lançada em junho de2008, com início da formação previsto a partir de 2009.

A ênfase na regulamentação da profissão e na formação técnica dos agentes comunitários de saúde, a partir da Emenda Constitucional no 51, de 2006, trouxe, para além do desafio da formação, 0 da forma de contratação. Esse desafio, não exclusivo da Saúde da Família, acentua o debate necessário acerca do emprego público no SUS.

\section{Desafio da APS como ordenadora do sistema}

A conformação de Territórios de Atenção à Saúde, a partir das equipes de Saúde da Família, deverá ser capaz dese relacionar com a urgência eemergência pela classificação deriscos eresolutividade dos agravos agudos de menor complexidade, a relação com as redes de serviços de saúde mental e das redes sociais, a integração com a vigilância em saúde, 0 acesso a exames complementares ea assistência farmacêutica. A APS deve ter maior poder e controle sobre os prestadores dos demais pontos da rede de atenção, inclusive com a utilização de ferramentas para avaliar a qualidade da atenção especializada, ambulatorial ou hospitalar, com vistas a aumentar a segurança dos pacientes. Essesterritóriostornamse, portanto, espaços do cuidado e da promoção da saúde, da intersetorialidade e da constante busca da qualidade de vida, da garantia do primeiro contato e do encaminhamento responsável para utilização coerente das tecnologias disponíveis nos serviços de referência especializada.

A importância da integralidade, de se ofertar serviços que estabeleçam o tratamento e recuperação dos doentes, mas que essencialmente promo- vam a saúde e previnam o adoecimento, inclusive evitando a utilização desnecessária das tecnologias, fez com que a Saúde da Família fosse campo das várias ações intersetoriais, junto ao M inistério do Desenvolvimento Social e Combateà Fome, com o acompanhamento das condicionalidades do Bolsa Família, ao M inistério da Educação, com o Saúde na Escola, M inistério da Justiça, com o PRONAS$\mathrm{Cl}$, M inistério do Desenvolvimento Agrário, com os Territórios da Cidadania, M inistério da Cultura, com a integração com os pontos de cultura ea criação da Rede Saúde e Cultura.

Avançamos assim, na possibilidade da saúde ser experimentada, pelosindivíduos, famílias e comunidades, como um "fenômeno clínico e sociológico, mas vivido cultural mente", utilizando o conceito de saúde proposto por M inayo. A busca da qualidade de vida para a população brasileira só é possível com ações que extrapolem nossa capacidade setorial, desde que asseguradas as melhores evidências na oferta de ações e serviços de saúde.

\section{Desafio da comunicação com a sociedade}

Há também, de forma imperiosa, a necessidade de aproximação com a sociedade brasileira formadora de opinião, que, na maior parte das vezes, desconhece esse modo de acessar as ações e serviços desaúde. Aquilo quenão éconhecido, não pode ser defendido pela população. Entendemos a atenção primária como capaz de conduzir a sociedade na definição dos seus direitos, incorporando os conceitos de empoderamento e capital social, ou seja, toda a sociedade brasileira recebendo o mesmo tipo de atenção à saúde.

Se há suficientes evidências que o cumprimento dos atributos dos serviços de APS - acesso e utilização, integralidade, Iongitudinalidade ecoordenação - estão associados com menor número de hospitalizações, menor número de consultas para um mesmo problema, menor quantidade de exames complementares, maior possibilidade de ações de prevenção, maior adesão a tratamentos, dietas, execução de exames e consultas de encaminhamentos e também maior satisfação da população, podemos inferir que cidadãos satisfeitos com os serviços que recebem defenderão o modelo público eaprovarão o financiamento necessário para sua manutenção.

0 ano de 2008 marcou outro importante marco da advocacy da APS no Brasil, que foi a III M ostra de Experiências em Saúde da Família, ocorrida em Brasília (DF), com a presença de mais de 7.400 pessoas. Foi uma iniciativa de reforço técnico epolí- 
tico da Saúdeda Família, bem como divulgou internacionalmente o Sistema Ú nico de Saúdee o nosso modelo deAPS. Essa construção éfruto do esforço e da perseverança de milhares de brasileiros que fazem a Saúde da Família no Brasil, trabalhadores, usuários, gestores, conselheiros de saúde, pesquisadores, alunos e professores das áreas de saúde, enfim todos os envolvidos no sonho de um sistema de saúde universal, integral e equânime para o Brasil. 0 compartilhamento de experiências e também das dificuldades enfrentadas por equipes, municípios, estados e diferentes países do mundo demonstra a capacidade de mobilização da APS. No Brasil, o potencial criativo avança para além das normas e diretrizes estabelecidas, resul- tando numa diversidade de experiências e resultados exitosos, que precisam ser traduzidos para a sociedade, por meio de todas as mídias: TV, rádio, internet, jornal, cinema e telefones, como parte do movimento nacional pela democratização e pelo direito à comunicação e informação.

$\mathrm{O}$ relatório anual da $\mathrm{OMS}^{9}$ - Primary $\mathrm{H}$ ealth Care, now more than ever - recém-publicado, reforça a APS como uma idéia-força após trinta anos de Alma-A ta e el ogia a experiência brasileira9. Estamos no caminho certo, vencer os desafios apontados é obrigação da sociedade brasileira na manutenção de sua mai or política de inclusão social, que é o Sistema Ú nico de Saúde, agora orientado pela atenção primária/Saúde da Família.

\section{Referências}

1. Brasil. Ministério da Saúde. Secretaria de Atenção À Saúde. Departamento de Atenção Básica.Política Nacional de Atenção Básica. 4ạ ed. Brasília : M inistério da Saúde; 2007.

2. Starfield B. Atenção Primária: equilíbrio entre necessidades de saúde, serviços e tecnologia. Brasília: UNESCO/M inistério da Saúde; 2002.

3. Elias PE, Ferreira CW, Alves MCG, Cohn A, Kishima V, Escrivão Jr. A, Gomes A, Bousquat A. Atenção Básica em Saúde: comparação entre PSF e UBS por estrato de exclusão social no município de São PauIo. Cien Saude Colet 2006; 11(3):633-641.

4. Facchini LA, Piccini RX, Tomasi E, Thumé E, Silveira DS, Siqueira FV, Rodrigues MA. Desempenho do PSF no Sul e no Nordeste do Brasil: avaliação institucional e epidemiológica da Atenção Básica à Saúde. Cien Saude Colet 2006;11(3):669-681.

5. Piccini RX, Facchini LA, Tomasi E, Thumé E, Silveira DS, Siqueira FV, Rodrigues MA. Necessidades de saúde comuns aos idosos: efetividade na oferta e utilização em atenção básica à saúde. Cien Saude Colet 2006; 11(3):657-667.
6. Macinko J, Guanais F, Souza F. An Evaluation of the Impact of the Family Health Program on Infant M ortality in Brazil, 1990-2002. Journal of Epidemiology and Community $\mathrm{H}$ ealth 2006;60:13-19.

7. Murray CJL, Laakso T, Shibuya K, Hill K, Lopez AD. Can we achieve Millenium Development Goal? N ew analysis of country trends and forecasts of under mortality to 2015. Lancet 2007; 370:1040-1504.

8. Brasil. M inistério da Saúde.Secretaria de Atenção à Saúde. Departamento de Atenção Básica. Saúde da Família no Brasil: uma análise de indicadores selecionados:1998-2006/2006. Brasília: M inistério da Saúde; 2006.

9. World Health Organization. The World Health Report 2008. Primary Health Care, now more than ever. Geneve: WHO; 2008.

Artigo apresentado em: 26/11/2008 Aprovado em 05/05/2009 Versão final apresentada em 10/05/2009 